\title{
Fusion of the dendritic cell-targeting chemokine MIP3a to melanoma antigen Gp100 in a therapeutic DNA vaccine significantly enhances immunogenicity and survival in a mouse melanoma model
}

\author{
James T. Gordy ${ }^{1}$, Kun Luo ${ }^{1}$, Hong Zhang ${ }^{1}$, Arya Biragyn ${ }^{2}$ and Richard B. Markham*
}

\begin{abstract}
Background: Although therapeutic cancer vaccines have been mostly disappointing in the clinic, the advent of novel immunotherapies and the future promise of neoantigen-based therapies have created the need for new vaccine modalities that can easily adapt to current and future developments in cancer immunotherapy. One such novel platform is a DNA vaccine fusing the chemokine Macrophage Inflammatory Protein-3a (MIP-3a) to an antigen, here melanoma antigen gp100. Previous published work has indicated that MIP-3a targets nascent peptides to immature dendritic cells, leading to processing by class I and II MHC pathways. This platform has shown enhanced efficacy in prophylactic melanoma and therapeutic lymphoma model systems.

Methods: The B16F10 melanoma syngeneic mouse model system was utilized, with a standard therapeutic protocol: challenge with lethal dose of B16F10 cells $\left(5 \times 10^{4}\right)$ on day 0 and then vaccinate by intramuscular electroporation with $50 \mathrm{\mu g}$ plasmid on days three, 10, and 17. Efficacy was assessed by analysis of tumor burden, tumor growth, and mouse survival, using the statistical tests ANOVA, mixed effects regression, and log-rank, respectively. Immunogenicity was assessed by ELISA and flow cytometric methods, including intracellular cytokine staining to assess vaccine-specific T-cell responses, all tested by ANOVA.

Results: We demonstrate that the addition of MIP3a to gp100 significantly enhances systemic anti-gp100 immunological parameters. Further, chemokine-fusion vaccine therapy significantly reduces tumor burden, slows tumor growth, and enhances mouse overall survival compared to antigen-only, irrelevant-antigen, and mock vaccines, with efficacy mediated by both CD4+ and CD8+ effector T cells. Antigen-only, irrelevant-antigen, and chemokine-fusion vaccines elicit significantly higher and similar CD4+ and CD8+ tumor-infiltrating lymphocyte (TIL) levels compared to mock vaccine. However, vaccine-specific CD8+ TILs are significantly higher in the chemokine-fusion vaccine group, indicating that the critical step induced by the fusion vaccine construct is the enhancement of vaccine-specific T-cell effectors.

(Continued on next page)
\end{abstract}

\footnotetext{
* Correspondence: Rmarkha1@jhu.edu

${ }^{1}$ The Department of Molecular Microbiology and Immunology, Johns Hopkins Bloomberg School of Public Health, 615 N. Wolfe Street, Baltimore, MD 21205, USA

Full list of author information is available at the end of the article
} International License (http://creativecommons.org/licenses/by/4.0/), which permits unrestricted use, distribution, and reproduction in any medium, provided you give appropriate credit to the original author(s) and the source, provide a link to the Creative Commons license, and indicate if changes were made. The Creative Commons Public Domain Dedication waiver (http://creativecommons.org/publicdomain/zero/1.0/) applies to the data made available in this article, unless otherwise stated. 
(Continued from previous page)

Conclusions: The current study shows that fusion of MIP3a to melanoma antigen gp100 enhances the immunogenicity and efficacy of a DNA vaccine in a therapeutic B16F10 mouse melanoma model. This study analyzes an adaptable and easily produced MIP3a-antigen modular vaccine platform that could lend itself to a variety of functionalities, including combination treatments and neoantigen vaccination in the pursuit of personalized cancer therapy.

Keywords: DNA Vaccine, MIP3a, MIP3alpha, or CCL20, B16 Melanoma, Gp100, Therapeutic cancer vaccine, Chemokine-antigen fusion, In vivo electroporation

\section{Background}

The recent therapeutic success of immunotherapies [1] and the identification of cancer neoantigens as potential therapeutic targets $[2,3]$ have generated renewed interest in the field of cancer vaccines. Although only one therapeutic cancer vaccine is currently FDA-approved (Sipuleucel-T [4]), hypothesized synergies between current and future immunotherapies [5] have increased the need for new vaccine platforms that can best address the new immunotherapeutic opportunities.

DNA vaccines offer many advantages as cancer therapies. They generate effector immunity from all three arms of the adaptive immune response, particularly including CD8+ T-cells [6]. They avoid the inclusion of extraneous and possible deleterious antigens that may be components of bacterial or viral-based vaccines [6]. They stimulate innate immunity and avoid issues of safety and practicality associated with various vectors [6]. They can also be readily adapted to novel or mutating antigenic targets, are stable at room temperature, and can be constructed quickly [6]. Clinical trials with a variety of antigens have demonstrated safety and immunogenicity of clinical DNA vaccines [7, 8]. However, initial trials for therapeutic DNA cancer vaccines have all shown limited effectiveness [9]. More recent advances in DNA vaccination modalities have rekindled interest in their potential efficacy for cancer therapy $[10,11]$. Of note, DNA vaccines have shown efficacy in animals, with three licensed for veterinary use [12-14].

One of the primary hurdles for DNA vaccines has been their limited potency in the clinical setting [6]. Novel approaches to in vivo DNA delivery are being developed to address this issue. In vivo electroporation has been shown in animal models to enhance the breadth and potency of elicited immune responses [15-18]. Mechanistic studies have shown electroporation increases DNA uptake, stimulates local inflammation at the vaccination site, and enhances amount of vaccine antigen produced in situ [19-21]. In vivo electroporation is currently being utilized in the veterinary clinic as a mode of introducing a hormone into pregnant sows [22] and is currently undergoing clinical trials [23, 24].

Additionally, investigators have been taking advantage of the inherent flexibility of DNA to add immunomodulators to the vaccine construct in order to enhance the efficiency of initiating a specific immune response. Many studies have focused on increasing productive contact of nascent vaccine antigens to antigen presenting cells (APCs), especially dendritic cells (DCs). One approach is to fuse antigens to cytokines such as GM-CSF that can stimulate the development, proliferation, and maturation of DCs and monocytes [25-27] or to chemokines like CCL5 [28], CCL19 [29], MIP3 $\alpha$ (also known as CCL20) [30-35], or other molecules [36-40] that can recruit and/or target nascent peptides to APCs. MIP3 $\alpha$ fusion vaccines have been shown to direct antigen to immature DCs via CCR6 and mediate antigen uptake in a fusion dependent manner [39], after which, antigens are cross presented by both MHC class I and II, activating significant responses from both CD4+ and CD8+ T cells [31-33].

In the current studies, a DNA vaccine administered by intramuscular electroporation with a construct fusing MIP3 $\alpha$ to the melanoma tumor-associated antigen gp100 has been analyzed in a therapeutic vaccination protocol utilizing the B16F10 melanoma mouse model system. MIP3 $\alpha$-antigen fusion DNA vaccine constructs have shown efficacy in a prophylactic melanoma model against gp100 [33], a therapeutic lymphoma model against oncofetal antigen (OFA) [31], and a prophylactic malaria model against circumsporozoite protein (CSP) [30]. Here we compare therapeutic MIP3 $\alpha$-gp100 vaccination to a construct with a mutated MIP3 $\alpha$ sequence that abrogates its function, effectively providing a gp100 antigen-only vaccine, and to a construct fusing the chemokine to an antigen irrelevant to this system, CSP. These experiments show that inclusion of functional MIP3 $\alpha$ in the vaccine construct used in the therapeutic protocol enhances immunogenicity, slows tumor growth, and significantly extends survival compared to antigenonly and irrelevant-antigen vaccinations.

\section{Methods}

\section{Animals and tumor model}

Five to six week old female C57BL/6 (H-2b) mice were purchased from Charles River Laboratories (Wilmington, MA) and maintained in a pathogen-free microisolation facility in accordance with the National Institutes of Health guidelines for the humane use of laboratory animals. All experimental procedures involving 
mice were approved by the IACUC of the Johns Hopkins University (Protocol number MO13H219 and MO16H85). B16F10 mouse melanoma cells were a generous gift from Dr. Arya Biragyn (NIH, Baltimore, MD). Six to eight week old mice were challenged in the left flank subcutaneously with a lethal dose $\left(5 \times 10^{4}\right.$ cells $)$ of B16F10 melanoma. Tumor size was recorded as square $\mathrm{mm}$, representing tumor length $\times$ width (opposing axes) measured by calipers every 1-3 days. Mice were kept in the study until one of the following occurred: mouse death, tumor size eclipsing $20 \mathrm{~mm}$ in any direction, or extensive tumor necrosis resulting in excessive bleeding.

\section{Plasmids and vaccination}

Vaccine consisted of purified plasmid DNA in endotoxinfree PBS. The plasmid encoded either MIP3 $\alpha$-gp100, MIP3 $\alpha$-CSP as described [30], or dMIP3 $\alpha$-gp100 fusion sequence as described [33]. dMIP3 $\alpha$-gp100 vaccine DNA is identical except for a point mutation in the chemokine changing a structurally necessary cysteine to serine (C6S), which abrogates chemokine functionality [33]. Vaccination plasmid was extracted from E. coli using Qiagen ${ }^{\circ}$ (Germantown, Md) EndoFree ${ }^{\odot}$ Plasmid Maxi and Giga Kits. Vaccine DNA purity, quality, and quantity were verified by gel electrophoresis, restriction enzyme analysis, Nanodrop ${ }^{\circ}$ spectrophotometry, and full insert sequencing. Mock vaccinations comprised of endotoxin-free PBS only. DNA injections were administered into the hind leg tibialis muscle. Immediately following injection, the muscle was pulsed using an ECM 830 Electro Square Porator ${ }^{\mathrm{mi}}$ with 2-Needle Array ${ }^{\mathrm{ma}}$ Electrode (BTX Harvard Apparatus ${ }^{\circ}$; Holliston, MA) under the following parameters: $106 \mathrm{~V} ; 20 \mathrm{~ms}$ pulse length; $200 \mathrm{~ms}$ pulse interval; 8 total pulses. Vaccinations of 50ug/dose were delivered at days noted in figure legends. Prophylactic efficacy of the vaccine was confirmed, replicating previously reported results in which DNA was delivered by gene gun [33] [Additional file 1]. Vaccine DNA was also confirmed to express specific protein after transfection into Hek-293 $\mathrm{T}$ cells [Additional file 2], as detected by Western blot analysis using antibodies targeting the myc tag present at the 3' end of the construct. As described by others, vaccination for the therapeutic model began on day three $[41,42]$.

\section{In cell ELISA}

Humoral immune responses to the vaccine were tested by an In-Cell ELISA assay to detect overall response to native B16F10 proteins, including gp100. The studies utilized the standard protocol for In-Cell ELISA from Abcam $^{\circ}$ (Cambridge, UK). In brief, wells of tissueculture treated 96-well plates were seeded with $5 \times 10^{4}$ B16F10 cells and were allowed to adhere for $3-4 \mathrm{~h}$ at $37^{\circ} \mathrm{C}$. Adherence was verified by microscopy before proceeding. Cells were fixed, incubated with serum or primary control antibody (rabbit anti-gp100 ab137078 [Abcam, Inc.; Cambridge, UK]) at varying dilutions overnight at $4{ }^{\circ} \mathrm{C}$, blocked with $2 \% \mathrm{BSA}$, and then incubated with peroxidase-conjugated goat anti-mouse IgG (serum) or goat anti-rabbit IgG(control) (Jackson ImmunoResearch Laboratories, West Grove, PA) at a dilution of 1:5000. Wells were developed for $1 \mathrm{~h}$ using $\mathrm{ABTS}^{\circ}$ ELISA HRP Substrate (KPL, Gaithersburg, MD). The data were collected using the Synergy $^{\text {mit }}$ HT (BioTek Instruments, Inc. Winooski, VT).

\section{Extraction of splenocytes and TILs}

Spleen and tumor cell suspensions were prepared by grinding sterile excised tissue between the frosted ends of microscope slides and then passing the tissue through a sterile $60 \mu \mathrm{M}$ mesh. Splenocytes were processed by lysing red blood cells and washing with sterile PBS. Tumor lysate was washed with sterile PBS, and tumorinfiltrating lymphocyte (TIL) fraction was enriched by Lympholyte ${ }^{\oplus} \mathrm{M}$ Cell Separation Media (Cedarlane ${ }^{\circ}$, Burlington, NC) according to the manufacturer's protocol. Prior to use all cells were counted by a $\mathrm{Z}^{\mathrm{m}}$ Coulter Counter ${ }^{\circ}$ (Beckman Coulter, Inc.; Brea, CA) and/or a hemocytometer with Gibco ${ }^{\text {tm }}$ Trypan Blue solution 0.4\% (Life Technologies, Carlsbad, CA).

\section{Intracellular cytokine staining and flow cytometry}

Enriched splenocytes or TILs were seeded onto Falcon ${ }^{\circ}$ Multiwell 24-well tissue culture treated plates (Corning, Inc.; Corning, NY) at $1 \times 10^{6}$ cells per well (or all cells if total is less) and stimulated for $3-4 \mathrm{~h}$ at $37{ }^{\circ} \mathrm{C}$ with known immunodominant gp100 $25-33$ (KVPRNQDWL) peptide or control HA (YPYDVPDYA) peptide (JHU School of Medicine Synthesis \& Sequencing Facility; Baltimore, MD) combined with Protein Transport Inhibitor Cocktail and costimulatory anti-CD28 and anti-CD49d agonizing antibodies (eBioscience, Inc. San Diego, Ca). Cells were collected, washed, fixed, permeabilized, and stained using standard laboratory protocols for intracellular staining. Fixation and permeabilization buffers from Mouse Regulatory $\mathrm{T}$ Cell Staining Kit \#2 (eBioscience, Inc. San Diego, Ca) were used. Stains utilized were the following antimouse mAbs: PercPCy5.5 conjugated anti-CD3, APCconjugated anti-IFN $\gamma$, FITC-conjugated anti-CD8, and PE-conjugated anti-CD4 (eBioscience, Inc. San Diego, CA). Utilized FACSCalibur ${ }^{\text {Tw }}$ and LSRII ${ }^{\mathrm{Tm}}$ Flow Cytometers (BD Biosciences, San Jose, CA). Flow Data analyzed by FlowJo Software (FlowJo, LLC Ashland, OR).

\section{Lymphocyte depletion}

To deplete the CD4+, CD8+, or both T cell subsets, immunized mice were injected i.p. with anti-CD4 (GK1.5), anti-CD8 (2.43), or both mAbs, which were generous 
gifts from Dr. Fidel Zavala (JHSPH, Baltimore, MD). Negative control vaccinated mice received isotype Rat IgG2b antibody against KLH (LTF-2) purchased from BioXCell (West Lebanon, NH). $100 \mu \mathrm{g}$ of antibody was given to each mouse i.p. on days $-1,0$, and 7 from tumor challenge. Depletion efficacy was tested on days 0 and 8 or 10 by two-color flow cytometry analysis of peripheral blood lymphocytes using a FACSCalibur ${ }^{\text {rm }}$ cytometer (BD Biosciences, San Jose, Ca) with FITC conjugated antimouse CD4 and APC-conjugated anti-mouse CD8 mAbs (eBioscience, Inc. San Diego, Ca).

\section{Statistics and availability of data}

Tumor size, immunologic, and flow cytometric analyses were statistically tested by one-way ANOVA with Bonferonni correction and/or Tukey's multiple comparisons test. Mouse survival studies were statistically tested by the log-rank test. Tumor time course regressions were analyzed by mixed effect regression models. STATA v11.2 (StataCorp, College Station, TX) and Prism 6 (GraphPad Software, Inc. San Diego, CA) were utilized for statistical analyses and figure creation. Significance level of $\alpha \leq 0.05$ was set for all experiments. The dataset supporting the conclusions of this article is included within the article's additional files [Additional file 3].

\section{Results}

\section{Systemic immune response}

To initially evaluate the immunogenicity of the DNA construct, systemic immune parameters were examined. Mice were vaccinated three times at 1 week intervals and then analyzed 2 weeks after the third immunization. The MIP3 $\alpha$-gp100 vaccine elicited significantly higher levels of B16F10-specific antibodies than antigen-only vaccine, denoted as dMIP3 $\alpha$-gp100 $(p=0.004)$, and mock vaccine $(p<0.001$; Fig. 1$)$. Interestingly, antigenonly vaccine had significantly higher B16F10-specific antibody levels than mock vaccine $(p=0.044)$.

As was the case for the antibody concentration, the antigen-only vaccine elicited a moderate vaccine-specific CD8+ T-cell response that significantly differed from the mock vaccination by both percentage $(p=0.030$; Fig. $1 \mathrm{~b})$ and total number $(p<0.001$; Fig. $1 c)$ of $\mathrm{CD} 8+\mathrm{T}$ cells reactive to the immunogenic gp100 $25-33$ peptide. The addition of MIP3 $\alpha$ to the vaccine significantly increased the percentage of $(p=0.049)$ and total number of $(p=$ 0.026 ) vaccine induced $C D 8+T$ cells compared to the antigen only vaccine, increasing the CD8+ $\mathrm{T}$ cell numbers by $46 \%$ (Fig. 1b-c). The MIP3 $\alpha$-gp100 vaccine elicited significantly higher percentages and numbers of vaccine-specific $\mathrm{CD} 8+\mathrm{T}$ cells compared to mock vaccination $(p<0.001$ for both; Fig. 1b-c).

\section{Therapeutic vaccination model}

The potential of this vaccine construct to be utilized in a therapeutic setting against a solid tumor was assessed,

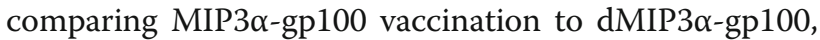
MIP3 $\alpha$-CSP, and PBS vaccines. A therapeutic regimen was developed with mice vaccinated on days three, 10 , and 17 post challenge with a lethal dose of B16F10 cells. Utilizing statistical mixed effects regression models, it was determined that the overall slope of the tumor growth regression line was reduced in the MIP3 $\alpha$-gp100 vaccinated group compared to the antigen-only vaccinated group by $48 \%(p=0.029)$, to the irrelevant-antigen group by $56 \%(p<0.001)$, and to the mock vaccine group by $63 \%(p<0.001)$, whereas mock, antigen-only, and irrelevant-antigen vaccines showed no significant differences to each other in slope (Fig. 2a). Slower overall growth also provides evidence that the differences seen in these experiments are not due to blocks to tumor transplantation.

In addition to tumor growth, the tumor burden of MIP3 $\alpha$-gp100 vaccine recipients proved to be significantly lower than mock vaccination at most time-points tested and significantly lower than antigen only vaccine on the critical day 14 time-point - the last time point before any mice were removed from the study. On day 14 post challenge, the average tumor size was reduced in the MIP3 $\alpha$-gp100 group by $51 \%$ compared to the antigen-only group $(p=0.004)$, by $54 \%$ compared to irrelevant-antigen group $(p=0.001)$, and by $55 \%$ compared to the mock group ( $p=0.001$; Fig. $2 b)$. Survival analysis mirrored tumor growth and burden analyses. MIP3 $\alpha$-gp100 vaccination significantly enhanced survival as compared to antigen-only $(p=0.017)$, irrelevantantigen $(p=0.021)$ and mock $(p<0.001)$ vaccines. MIP3 $\alpha$-gp100 vaccination enhanced median survival by $10 \%, 24 \%$ and $24 \%$ compared to antigen-only, irrelevantantigen, and mock vaccinations, respectively (Fig. 3). Antigen-only, irrelevant-antigen, and mock vaccinations did not have significantly different survival curves compared to each other (Fig. 3).

\section{T-cell subset depletion}

To determine if effector T-cells played a role in mediating this enhanced protection, and, if so, which subsets might be involved, groups of mice were vaccinated three times over 3 weeks to develop vaccine-specific effector responses and then were challenged with tumor under differing depletion conditions: depleting CD4+, CD8+, both $\mathrm{CD} 4+$ and $\mathrm{CD} 8+$, and no depletion of $\mathrm{T}$ cells. Figure 4a shows representative flow cytometric analysis of depletion efficacy. In a mouse lymphoma model, a similar MIP3 $\alpha$-OFA vaccine showed the CD8+ T-cell effector response to be essential for protection with the CD4+ T-cell effector response being expendable [31]. 

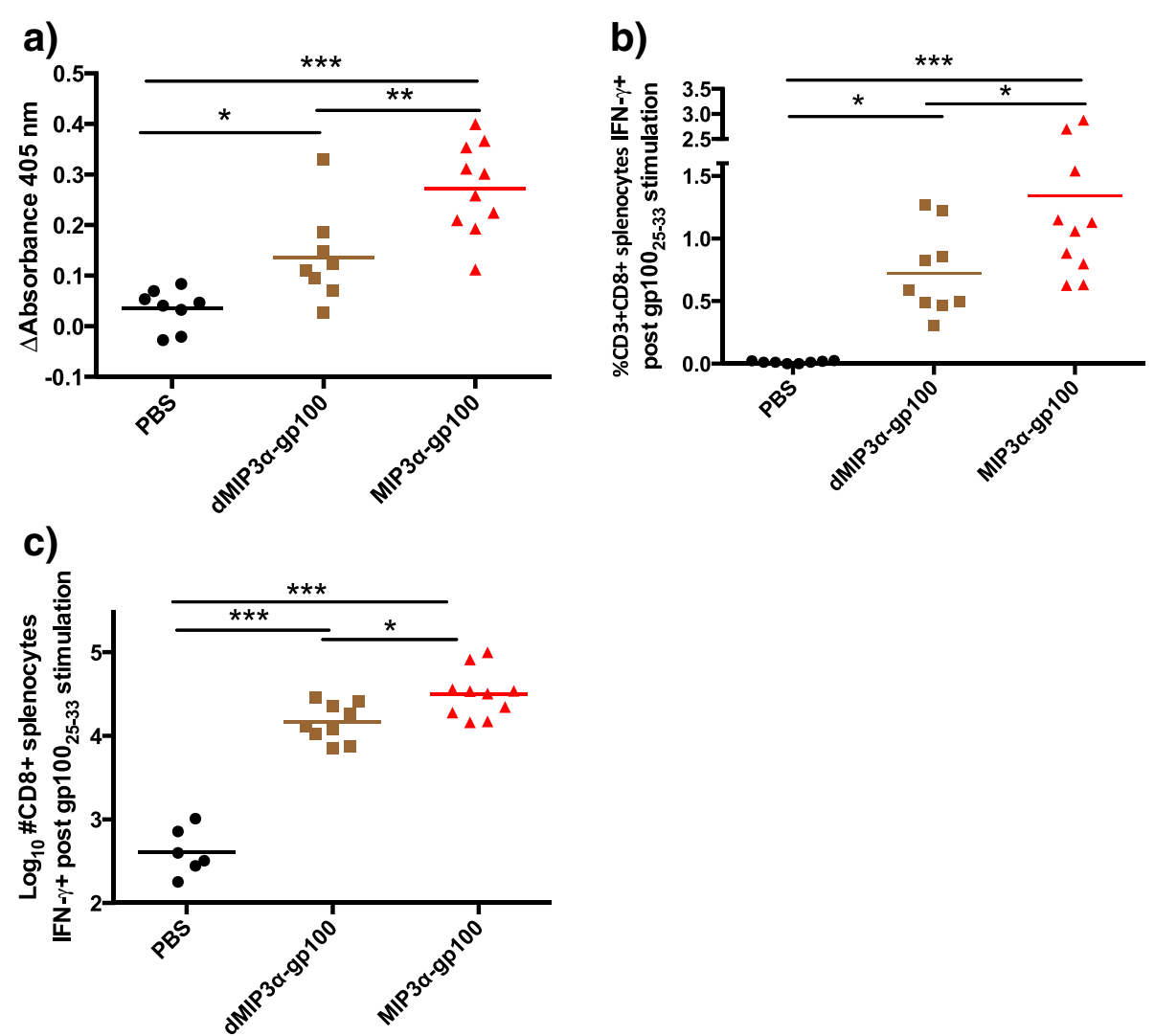

Fig. 1 Systemic immune parameters of vaccine groups in prophylactic vaccination setting. Mice were vaccinated three times at 1 week intervals with endotoxin-free PBS, dMIP3a-gp100, and MIP3a-gp100 fusion vaccine. Analysis occurred 2 weeks post third vaccination. Data represent two independent experiments with 3-5 mice per group per experiment. a Analysis of relative antibody production against B16F10 cells. In-Cell ELISA performed utilizing fixed B16F10 cells as antigens. Experimental data are shown at a 1:2000 serum dilution after 30-min colorimetric development. Absorbance values from pre-immune mice were subtracted from post immune mice to obtain the delta absorbance. All groups were significantly different from each other by ANOVA. $\mathbf{b}-\mathbf{c}$ Analysis of splenic CD8+ T cells reactive to ex vivo stimulation by gp $100_{25-33}$ peptide. Activation was signaled by cytoplasmic IFN- $\gamma$ accumulation as measured by Intracellular Cytokine Staining Flow Cytometry. Panel b shows the data as percentage of CD3+ splenocytes. Panel c estimates the total number of reactive CD3 + CD8+ splenocytes by extrapolating flow cytometric data to measured splenic total cell counts. For both panels, all groups differ significantly from each other, as determined by by ANOVA, ${ }^{*} p<0.05$,

${ }^{* *} p<0.01,{ }^{* * *} p<0.001$

However, in this melanoma solid tumor model, depleting CD4+ or CD8+ T-cells individually show a similar phenotype as the isotype depletion control. Single depletions have similar tumor growth rates and tumor sizes compared to isotype depletion (Fig. 4b-c). Importantly, depleting both subsets of T-cells simultaneously provided a phenotype similar to the unvaccinated control in those same analyses (Fig. 4b-c).

\section{Tumor infiltrating lymphocytes}

It has been documented that presence and activity of tumor infiltrating lymphocytes (TILs) can correlate with anti-tumor responses in melanoma patients [43]. The intratumoral characteristics of MIP3 $\alpha$-antigen vaccine responses have not previously been documented. Utilizing a therapeutic vaccination protocol as outlined, total CD4+ and CD8+ TILs were harvested 1 week after the second vaccination, counted by flow cytometry, and normalized by tumor size. Surprisingly, MIP3 $\alpha$-gp 100 , irrelevant-antigen, and antigen-only vaccines all induced significantly higher CD8+ TIL (all $p<0.001$ ) and CD4+ TIL (all $p<0.01$ ) responses compared to mock vaccine and were at similar levels to each other (Fig. 5a-b). However, antigen-only and irrelevant-antigen vaccines did not provide clinically relevant responses, not differing significantly from the negative control group in tumor growth, size, and survival (Figs. 2 and 3). In this system, TIL levels themselves appear not to correlate with protection. In addition, vaccine did not significantly alter levels of tumor-infiltrating CD25 + Foxp3+ regulatory CD4+ T-cells (Data not shown).

Finally, the levels of CD8+ TILs that secrete IFN- $\gamma$ upon stimulation with immunodominant gp $100_{25-33}$ vaccine antigen were assessed. Antigen-only vaccine 

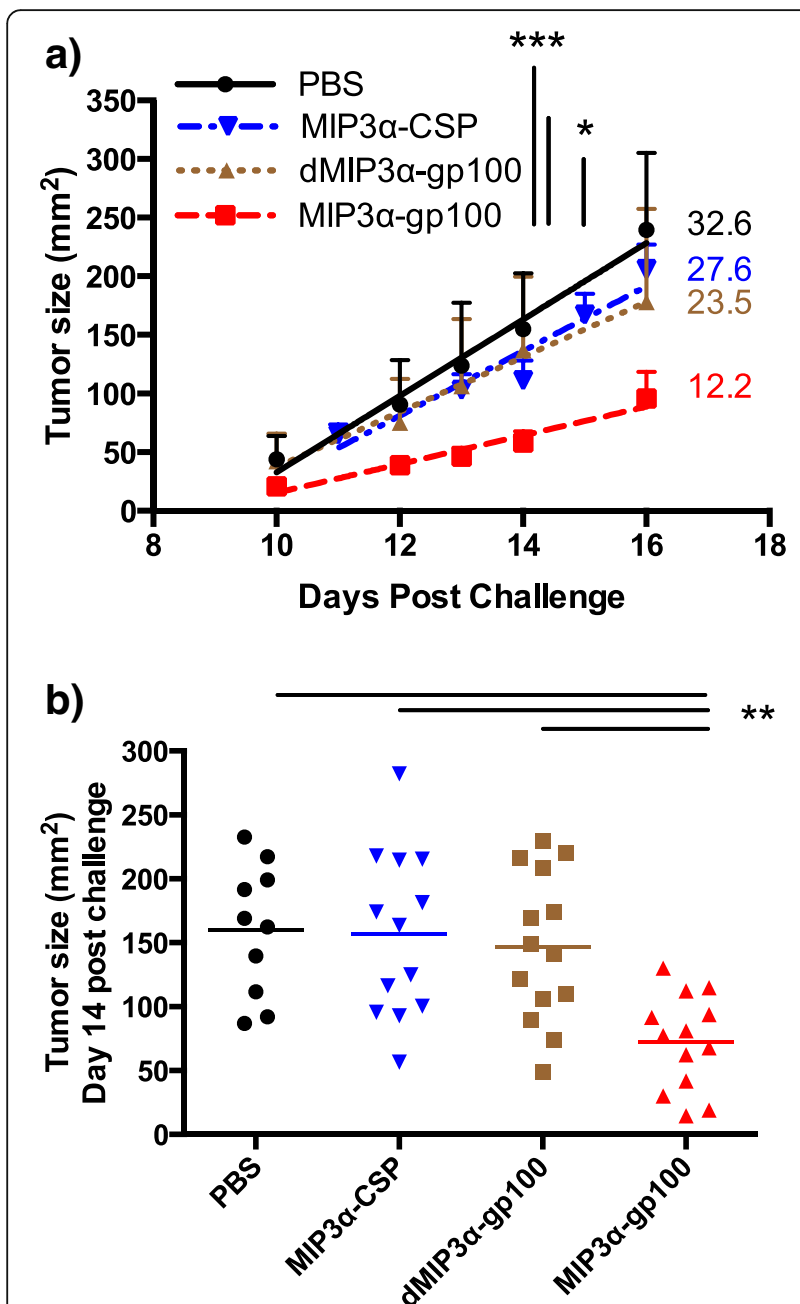

Fig. 2 Vaccine effects on tumor growth in therapeutic model. Vaccinations occurred on days 3, 10, and 17 post challenge. a Tumor growth rate was assessed between days 10 and 16, day 10 being the point at which tumor growth of the negative control group began accelerating and day 16 being the point at which mice began to be censored due to endpoints being reached. The graph shows one representative experiment of two, five to seven mice per group and includes linear regression lines and slopes. The slope of tumor growth among recipients of MIP3a-gp100 vaccine differed significantly from dMIP3a-gp100, MIP3a-CSP, and mock PBS vaccination, as evaluated using a statistical mixed effects regression model. The groups receiving dMIP3a-gp100 and MIP3a-CSP did not differ significantly compared to each other or to the group receiving mock vaccination. Error bars represent standard error. b Tumor size at day 14 post challenge, the last point before any mice were removed from experiments. The data are representative of two experiments, with 5-8 mice per group per experiment. MIP3a-gp100 vaccine recipients had significantly smaller tumors compared to dMIP3agP100, MIP3a-CSP, and mock PBS vaccinated mice, as determined by ANOVA. dMIP3a-gp100 and MIP3a-CSP were not significantly different from each other or from mock. ${ }^{*} p<0.05,{ }^{* *} p<0.01,{ }^{* * *} p<0.001$

induced a moderate response, with significantly higher gp100 $25-33$-reactive CD8+ TILs by percentage $(p<0.01)$ and normalized total numbers $(p<0.05)$ compared to

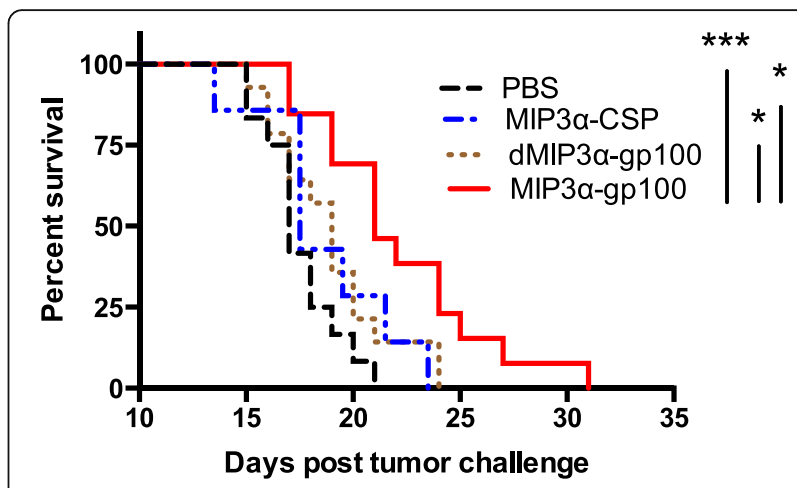

Fig. 3 Vaccine effects on mouse survival in a therapeutic model. Vaccinations occurred on days 3, 10, and 17 post challenge. Mice were removed from the study at the following endpoints: death, tumor size surpassing $2 \mathrm{~cm}$ in any dimension, or excessive tumor bleeding and ulceration. Data representative of two experiments, 5-8 mice per group per experiment. Mice in the MIP3a-gp100 vaccine group exhibit significantly enhanced survival compared to the dMIP3a-gp100, MIP3a-CSP, and mock PBS vaccination groups by the log-rank test. dMIP3a-gp100 and MIP3a-CSP did not differ significantly from each other or from mock. ${ }^{*} p<0.05,{ }^{* *} p<0.01,{ }^{* * *} p<0.001$

the PBS and irrelevant-antigen vaccinated negative control groups (Fig. 6a-b). MIP3 $\alpha$-gp100 vaccination significantly enhanced the percentage $(p<0.01)$ and normalized numbers $(p<0.05)$ of gp100 $25-33$-reactive CD8+ TILs compared to antigen-only vaccine and compared to irrelevantantigen $(p<0.001)$ and mock $(p<0.001)$. Although the two vaccines elicit a similar number of total TILs, the MIP3 $\alpha$-gp100 vaccine elicits a more robust vaccinespecific effector TIL response that correlates with the enhancement of tumor suppression and mouse survival seen.

\section{Discussion}

Our data demonstrate that the addition of the chemokine MIP3 $\alpha$ to the gp100 DNA vaccine construct enhanced vaccine immunogenicity and therapeutic potential. Although the antigen-only vaccine elicited a significant anti-gp100 immune response compared to the mock vaccine, when utilized as a therapy, only the MIP3 $\alpha$-gp100 vaccine slowed tumor growth and enhanced mouse survival. Further, MIP3 $\alpha$ fused to irrelevant antigen CSP showed no anti-tumor activity, despite the previously demonstrated ability of the CSP construct to function as a highly efficacious vaccine for preventing malaria in a mouse model system [30]. Previous studies have conclusively shown that MIP3 $\alpha$ must be fused to its antigen in order to enhance immunogenicity [39].

As has been shown in vitro [32, 33], MIP3 $\alpha$-gp100 vaccine directs the antigen in such a way that both CD4+ and CD8+ effector T-cells can be activated. In this study, $\mathrm{T}$-cells were depleted after a prophylactic vaccination regimen in order to selectively deplete vaccine-specific 
a)

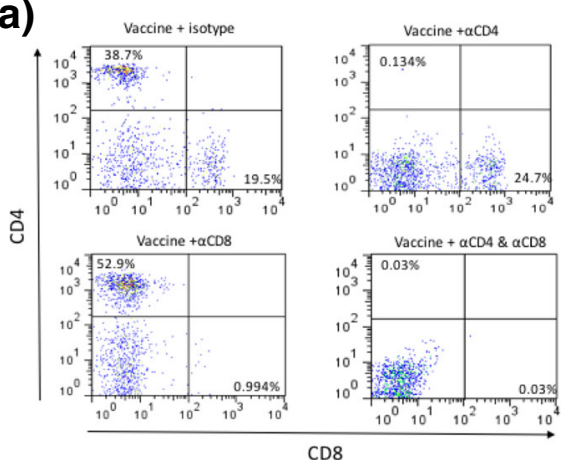

c)

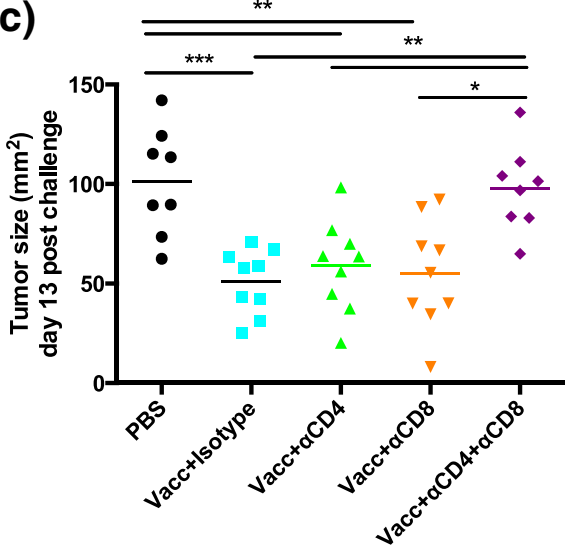

b)

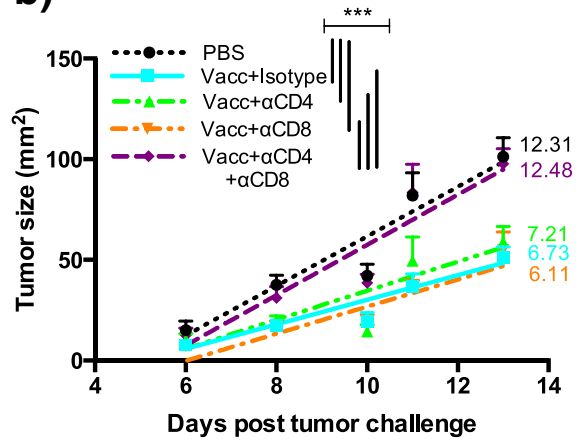

Fig. 4 Vaccine effector T-cell responses analyzed by subset depletions. Mice were vaccinated three times at 1 week intervals and then challenged with a lethal dose $\left(5 \times 10^{4}\right)$ of B16F10 cells. T-cell subsets were depleted one day prior to challenge, on day of challenge, and 7 days post challenge. Quality of the depletions was assessed by flow cytometric analysis of peripheral blood lymphocytes on days 0 and 8 or 10 . a Representative flow cytometry plots shown depicting CD4 and CD8 expression gated on overall lymphocytes from blood collected at day 10 post challenge. $\mathbf{b}$ Tumor growth regression plot from day 6 to 13 post challenge, assessed by mixed effects regression. c Tumor size at day 13 post challenge, with significance assessed by ANOVA. All data are from two independent experiments of 4-5 mice per group. ${ }^{*} p<0.05,{ }^{* *} p<0.01,{ }^{* * *} p<0.001$

effector cells and not disturb the immune activation phase of the vaccine response. If CD4+ T-cells were depleted in a therapeutic study, one would not know if the effect was due to lack of CD4+ anti-tumor effector response or due to lack of CD4+ T-cell help in the activation of a vaccinespecific CD8+ T-cell response.

Depletion of either the CD4+ or CD8+ effector T-cell population showed a protection phenotype similar to the non-depleted vaccine group, while depletion of both led to no protection, similar to that observed with mock vaccination. The lack of protection seen in the double depletion group provides evidence that antibodies elicited by the vaccine do not provide significant anti-tumor immunity on their own. Large tumor size outliers in both single depletion groups suggest that some proportion of the mice are reliant on the depleted subset for protection, but the overall groups either utilize both effector subsets relatively equally or one is able to compensate for lack of the other when necessary. The roles and mechanisms of tumor infiltrating effector CD4+ TILs are complex and still being defined [44], and therefore the intriguing finding of effector CD4+ T cells providing therapeutic efficacy in the absence of CD8+ T cells will be the subject of future work.

Finally, the data show that the therapeutic protection phenotype provided by MIP3 $\alpha$ did not correlate with overall TILs, but did correlate with gp $100_{25-33}$ vaccine peptide-reactive CD8+ TILs, elucidating that the immune activity of and not the quantity of the TILs correlates with therapeutic efficacy.

Vaccine efficacy depends on identification of appropriate target antigens, deliverance of those antigens in a form that elicits a relevant immune response, administration of vaccine by a route that brings it into contact with the critical immune cells, and selection of effective adjuvants/immunomodulators. For DNA vaccines, addition of MIP3 $\alpha$ to circumsporozoite protein (CSP) with vaxfectin adjuvant [30] creates a robust, protective antibody response against malaria, addition of MIP3 $\alpha$ to oncofetal antigen (OFA) given by gene gun creates a therapeutic response against lymphoma mediated by CD8+ T-cells [31], and as reported here, addition of 


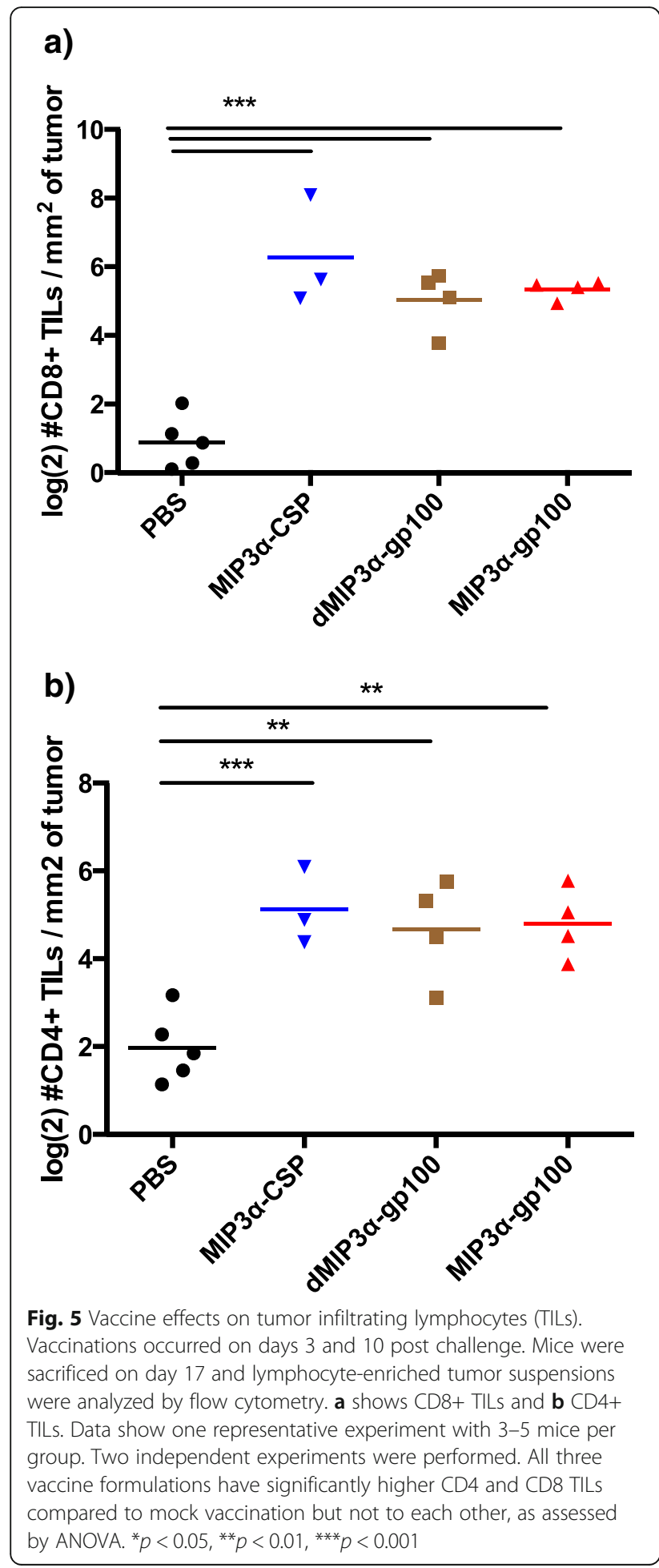

MIP3 $\alpha$ to gp100 given by intramuscular electroporation creates a therapeutic response against melanoma mediated by both CD4+ and CD8+ effector T-cells. All of these experiments have shown responses to be significantly enhanced by the chemokine in different contexts. Co-administration of MIP3 $\alpha$ can enhance vaccine

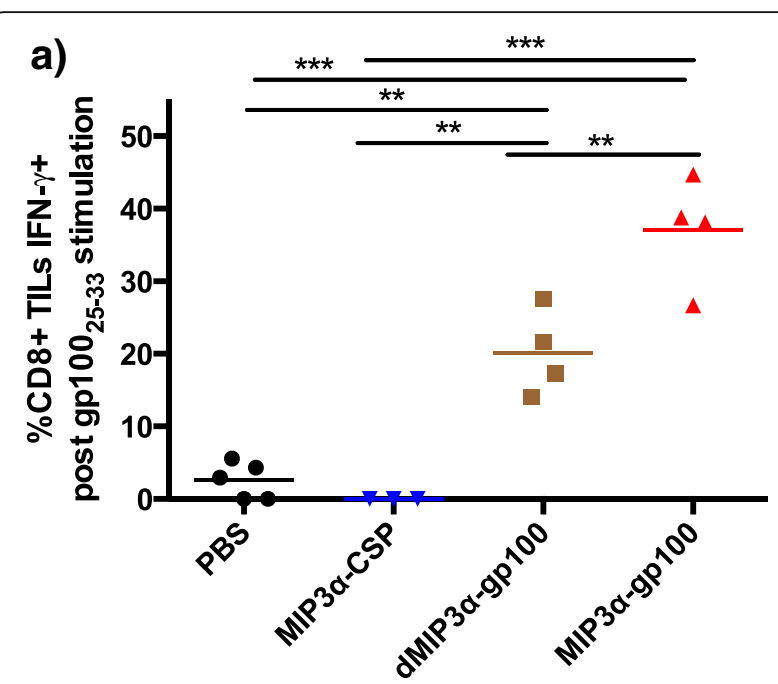

b)

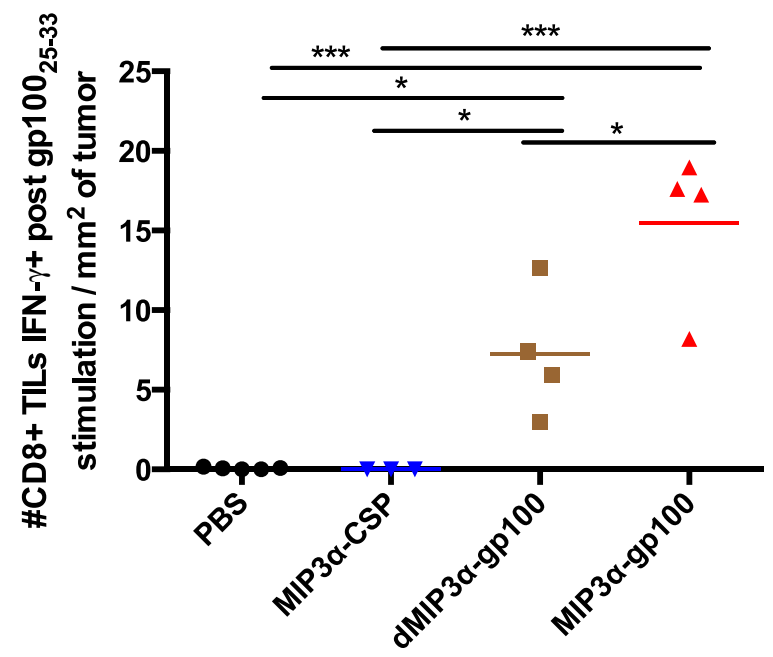

Fig. 6 Vaccine-specific CD8+ T-cell tumor infiltrate analysis. Vaccinations occurred on days 3 and 10 post challenge. Mice were sacrificed on day 17 and lymphocyte-enriched tumor suspensions were collected. CD8+

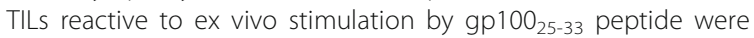
delineated by Intracellular Cytokine Staining Flow Cytometry measuring cytoplasmic IFN- $\gamma$ accumulation post stimulation. a Percentage of CD8+ TILs reactive to antigen. $\mathbf{b}$ Estimated total number of reactive CD8+ TILs normalized to tumor size. All groups were significantly different from each other by ANOVA except for the comparison of PBS to MIP3a-CSP. HA irrelevant negative peptide and PMA/ionomycin positive controls confirmed the protocol validity (data not shown). Data are from one of two representative experiments with 3-5 mice per group. ${ }^{*} p<0.05$, ${ }^{* *} p<0.01,{ }^{* * *} p<0.001$

responses by enhanced DC recruitment [45]. However, our previous studies have indicated that in the context of a DNA fusion vaccine, MIP3 $\alpha$ is acting by directing nascent expressed protein antigens to DCs, not by recruiting DCs in vivo [30]. Therefore, we hypothesize that in this context, the pro-inflammatory response elicited by electroporation serves as the adjuvant that 
recruits DCs to the vaccine site [19-21]. The MIP3 $\alpha$ fused to gp100 then increases the efficiency of nascent vaccine protein uptake into infiltrating immature dendritic cells, resulting in enhanced downstream effector responses. This research provides further evidence for the utility of adding chemokine immunomodulators to vaccine constructs within any immunological context.

A primary strength of this DNA vaccine system is its modularity and ease of construction. This study shows that taking the gp100 antigen that induces a specific albeit not therapeutically relevant response on its own can become therapeutically relevant simply by fusing it to MIP3 $\alpha$. This observation raises the possibility that the response to more immunogenic antigens could be even further enhanced by the addition of MIP3 $\alpha$. A burgeoning new field in cancer vaccinology is the utilization of cancer-specific neoantigens as better vaccine targets that are not subject to T-cell central tolerance restrictions [3]. Our modular DNA vaccine could easily and rapidly be constructed to utilize neoantigens as they are discovered in real time. Testing the principle of this idea will be the subject of future studies, utilizing now delineated immunogenic neoantigens found in the B16F10 cell line [46]. In addition to neoantigens, future studies will also examine the efficacy of this vaccine system with other solid tumor models, in combination with current treatments such as immune checkpoint blockade, and in combination with novel immunomodulators.

\section{Conclusions}

In conclusion, our data show that addition of MIP3 $\alpha$ enhances the immunogenicity and efficacy of a therapeutic vaccine against the aggressive solid tumor, B16F10 mouse melanoma. The addition of MIP3 $\alpha$ to therapeutic vaccines could present a useful strategy to enhance the responses of currently studied vaccines. Furthermore, the modularity of the plasmid provides a realistic platform for creating neoantigen vaccines in a clinically relevant time frame. These findings show that MIP3 $\alpha$ can be a plug and play addition to the cancer immunologist's vaccine toolbox that deserves further testing to determine the true potential of this novel design.

\section{Additional files}

Additional file 1: Figure S1. Prophylactic vaccination protection confirmation. Mice were vaccinated three times over 2 week intervals with PBS or $50 \mu \mathrm{g} \mathrm{MIP3a-gp100} \mathrm{by} \mathrm{i.m.} \mathrm{electroporation.} \mathrm{Mice} \mathrm{were}$ challenged with a lethal dose of B16F10 $\left(5 \times 10^{4}\right) 2$ weeks after the third immunization. Tumor time course was tracked and analyzed by linear regression models. Tumor growth was found to be significantly reduced $(p<0.001)$, replicating prior published data. Data represent one experiment with 5-6 mice per group. (DOCX $97 \mathrm{~kb}$ )

Additional file 2: Figure S2. Vaccine peptide production in mammalian cell culture system. Different lanes represent different DNA preparations, with Mock being untransfected HEK-293 T cells. Weights in kDa of the ladder bands are noted. Full length construct is estimated to be $40 \mathrm{kDa}$, consistent with primary band below. (DOCX $169 \mathrm{~kb}$ )

Additional file 3: Dataset. Excel spreadsheet of dataset used in this publication. This file includes all of the data points shown in figures of this study. (XLSX $48 \mathrm{~kb})$

\section{Abbreviations}

APC: Antigen presenting cell; B16F10: Mouse melanoma model; CSP: Circumsporozoite protein from malaria-causing parasite Plasmodium falciparum; DC: Dendritic cell; dMIP3a: Dysfunctional Macrophage Inflammatory Protein-3a. When utilized as a vaccine with gp100, it can be referred to as 'antigen-only vaccine'; GP100: Glycoprotein 100, common melanoma differentiation antigen and vaccine target; MHC: Major histocompatibility complex; MIP3a: Macrophage Inflammatory Protein-3a

\section{Acknowledgements}

We would like to acknowledge Dr. Fidel Zavala (Johns Hopkins School of Public Health, Baltimore, MD) for his generous gift of T-cell depletion antibodies. We would also like to acknowledge Dr. TC Wu (Johns Hopkins School of Medicine, Baltimore, MD) for allowing us to utilize his electroporator. We would like to acknowledge support for the statistical analysis from the National Center for Research Resources and the National Center for Advancing Translational Sciences (NCATS) of the National Institutes of Health through Grant Number 1UL1TR001079, and more specifically Carol Thompson of the Johns Hopkins Biostatistics Center.

\section{Funding}

We have no funding sources to disclose.

Availability of data and materials

As reported in the Methods, the dataset supporting the conclusions of this article is included within the article's additional files [Additional file 3].

\section{Authors' contributions}

JG performed, designed, and analyzed all the experiments and was the primary author of the manuscript. KL provided assisted with design and implementation of the mouse studies. $\mathrm{HZ}$ provided scientific direction, expertise, and help in data analysis. $A B$ contributed to the conception and design of these experiments. RM contributed to the conception, design, analysis of data, and the writing of the manuscript. All authors read and approved the manuscript.

\section{Competing interests}

J. Gordy and R. Markham are inventors on pending patents using the vaccine platform described in this manuscript. R. Markham has equity interest in a company that has rights to this vaccine platform. The authors declare that they have no competing interests.

\section{Ethics approval}

As reported in the Methods, mice were purchased from Charles River Laboratories (Wilmington, MA) and maintained in a pathogen-free microisolation facility in accordance with the National Institutes of Health guidelines for the humane use of laboratory animals. All experimental procedures involving mice were approved by the IACUC of the Johns Hopkins University (Protocol numbers $\mathrm{MO} 13 \mathrm{H} 219$ and $\mathrm{MO} 16 \mathrm{H} 85$ ).

\section{Author details}

${ }^{1}$ The Department of Molecular Microbiology and Immunology, Johns Hopkins Bloomberg School of Public Health, 615 N. Wolfe Street, Baltimore, MD 21205, USA. ${ }^{2}$ Immunoregulation Section, Laboratory of Molecular Biology and Immunology, National Institute on Aging, National Institutes of Health, 251 Bayview, Blvd, Suite 100, Baltimore, MD 21224, USA.

Received: 23 May 2016 Accepted: 7 November 2016 Published online: 20 December 2016 


\section{References}

1. Eggermont AMM, Maio M, Robert C. Immune checkpoint inhibitors in melanoma provide the cornerstones for curative therapies. Semin Oncol. 2015;42:429-35.

2. Overwijk W, Wang E, Marincola F, Rammensee H-G, Restifo N. Mining the mutanome: developing highly personalized Immunotherapies based on mutational analysis of tumors. J Immuno Ther Cancer. 2013;1:11.

3. Hacohen N, Fritsch E, Carter T, Lander E. Getting personal with neoantigenbased therapeutic cancer vaccines. Cancer Immunol Res. 2013;1:11-5.

4. Small EJ, Schellhammer PF, Higano CS, Redfern CH, Nemunaitis J J, Valone FH, Verjee SS, Jones LA, Hershberg RM. Placebo-controlled phase III trial of immunologic therapy with sipuleucel-T (APC8015) in patients with metastatic, asymptomatic hormone refractory prostate cancer. J Clin Oncol. 2006;24:3089-94.

5. Ali OA, Lewin SA, Dranoff G, Mooney DJ. Vaccines combined with immune checkpoint antibodies promote cytotoxic T-cell activity and tumor eradication. Cancer Immunol Res. 2016:4:95-100.

6. Liu M. DNA vaccines: an historical perspective and view to the future. Immunol Rev. 2011;239:62-84.

7. Tagawa S, Lee P, Snively J, Boswell W, Ounpraseuth S, Lee S, Hickingbottom B, Smith J, Johnson D, Weber J. Phase I study of intranodal delivery of a plasmid DNA vaccine for patients with Stage IV melanoma. Cancer. 2003;98:144-54.

8. Conry RM, Curiel DT, Strong TV, Moore SE, Allen KO, Barlow DL, Shaw DR, LoBuglio AF. Safety and immunogenicity of a DNA vaccine encoding carcinoembryonic antigen and hepatitis B surface antigen in colorectal carcinoma patients. Clin Cancer Res. 2002;8:2782-7.

9. Liu MA, Ulmer JB. Human clinical trials of plasmid DNA vaccines. Adv Genet. 2005;55:25-40.

10. Senovilla L, Vacchelli E, Garcia P, Eggermont A, Fridman W, Galon J, Zitvogel L, Kroemer G, Galluzzi L. Trial watch. Oncolmmunology. 2013;2:e23803.

11. Rice J, Ottensmeier C, Stevenson F. DNA vaccines: precision tools for activating effective immunity against cancer. Nat Rev Cancer. 2008;8:108-20.

12. Davis BS, Chang GJ, Cropp B, Roehrig JT, Martin DA, Mitchell CJ, Bowen R, Bunning ML. West Nile virus recombinant DNA vaccine protects mouse and horse from virus challenge and expresses in vitro a noninfectious recombinant antigen that can be used in enzyme-linked immunosorbent assays. J Virol. 2001;75:4040-7.

13. Anderson ED, Mourich DV, Fahrenkrug SC, LaPatra S, Shepherd J, Leong JA. Genetic immunization of rainbow trout (Oncorhynchus mykiss) against infectious hematopoietic necrosis virus. Mol Marine Biol Biotechnol. 1996;5: 114-22.

14. Bergman PJ, McKnight J, Novosad A, Charney S, Farrelly J, Craft D, Wulderk M, Jeffers Y, Sadelain M, Hohenhaus AE, Segal N, Gregor P, Engelhorn M, Riviere I, Houghton AN, Wolchok JD. Long-term survival of dogs with advanced malignant melanoma after DNA vaccination with xenogeneic human tyrosinase: a phase I trial. Clin Cancer Res. 2003;9:1284-90.

15. Chen J, Fang F, Li X, Chang H, Chen Z. Protection against influenza virus infection in BALB/C mice immunized with a single dose of neuraminidaseexpressing DNAs by electroporation. Vaccine. 2005;23:4322-8.

16. Medi BM, Hoselton S, Marepalli RB, Singh J. Skin targeted DNA vaccine delivery using electroporation in rabbits. I: efficacy. Int J Pharm. 2005;294:53-63.

17. Otten G, Schaefer M, Doe B, Liu H, Srivastava I, Zur Megede J, O'Hagan D, Donnelly J, Widera G, Rabussay D, Lewis MG, Barnett S, Ulmer JB. Enhancement of DNA vaccine potency in rhesus macaques by electroporation. Vaccine. 2004;22:2489-93.

18. Tollefsen S, Vordermeier M, Olsen I, Storset AK, Reitan LJ, Clifford D, Lowrie DB, Wiker HG, Huygen K, Hewinson G, Mathiesen I, Tjelle TE. DNA injection in combination with electroporation: a novel method for vaccination of farmed ruminants. Scand J Immunol. 2003;57:229-38.

19. Ahlén G, Söderholm J, Tjelle T, Kjeken R, Frelin L, Höglund U, Blomberg P, Fons $M$, Mathiesen I, Sällberg M. In vivo electroporation enhances the immunogenicity of hepatitis C virus nonstructural 3/4A DNA by increased local DNA uptake, protein expression, inflammation, and infiltration of CD3+ T cells. J Immunol. 2007;179:4741-53.

20. Liu J, Kjeken R, Mathiesen I, Barouch D. Recruitment of antigen-presenting cells to the site of inoculation and augmentation of human immunodeficiency virus type 1 DNA vaccine immunogenicity by in vivo electroporation. J Virol. 2008;82:5643-9.

21. Babiuk S, Baca-Estrada ME, Foldvari M, Middleton DM, Rabussay D, Widera G, Babiuk LA. Increased gene expression and inflammatory cell infiltration caused by electroporation are both important for improving the efficacy of DNA vaccines. J Biotechnol. 2004;110:1-10.

22. Person R, Bodles-Brakhop AM, Pope MA, Brown PA, Khan AS, Draghia-Akli R. Growth hormone-releasing hormone plasmid treatment by electroporation decreases offspring mortality over three pregnancies. Mol Ther. 2008;16:1891-7.

23. Vasan S, Hurley A, Schlesinger SJ, Hannaman D. In vivo electroporation enhances the immunogenicity of an HIV-1 DNA vaccine candidate in healthy volunteers. PLoS One. 2011;6:e19252.

24. Low L, Mander A, McCann K, Dearnaley D. DNA vaccination with electroporation induces increased antibody responses in patients with prostate cancer. Hum Gene Ther. 2009;20:1269-78.

25. Loudon PT, Yager EJ, Lynch DT, Narendran A, Stagnar C, Franchini AM, Fuller JT, White PA, Nyuandi J, Wiley CA, Murphey-Corb M, Fuller DH. GMCSF increases mucosal and systemic immunogenicity of an H1N1 influenza DNA vaccine administered into the epidermis of non-human primates. PLoS One. 2010;5:e11021.

26. Zheng Q, Fan D, Gao N, Chen H, Wang J, Ming Y, Li J, An J. Evaluation of a DNA vaccine candidate expressing prM-E-NS1 antigens of dengue virus serotype 1 with or without granulocyte-macrophage colony-stimulating factor (GM-CSF) in immunogenicity and protection. Vaccine. 2011;29:763-71.

27. Rodríguez AM, Pascutti MFF, Maeto C, Falivene J, Holgado MPP, Turk G, Gherardi MMM. IL-12 and GM-CSF in DNA/MVA immunizations against HIV1 CRF12_BF Nef induced T-cell responses with an enhanced magnitude, breadth and quality. PLoS One. 2012;7:e37801.

28. Lapteva N, Huang XF. CCL5 as an adjuvant for cancer immunotherapy. Expert Opin Biol Ther. 2010;10:725-33.

29. Nguyen-Hoai T, Hohn O, Vu MD, Baldenhofer G, Sayed Ahmed MS, Dörken B, Norley S, Lipp M, Pezzutto A, Westermann J. CCL19 as an adjuvant for intradermal gene gun immunization in a Her2/neu mouse tumor model: improved vaccine efficacy and a role for B cells as APC. Cancer Gene Ther. 2012;19:880-7

30. Luo K, Zhang H, Zavala F, Biragyn A, Espinosa D, Markham R. Fusion of antigen to a dendritic cell targeting chemokine combined with adjuvant yields a malaria DNA vaccine with enhanced protective capabilities. PLoS One. 2014;9:e90413.

31. Biragyn A, Schiavo R, Olkhanud P, Sumitomo K, King A, McCain M, Indig FE, Almanzar G, Baatar D. Tumor-associated embryonic antigen-expressing vaccines that target CCR6 elicit potent CD8+ T cell-mediated protective and therapeutic antitumor immunity. J Immunol. 2007;179:1381-8.

32. Biragyn A, Ruffini P, Coscia M, Harvey L, Neelapu S, Baskar S, Wang J-M, Kwak L. Chemokine receptor-mediated delivery directs self-tumor antigen efficiently into the class II processing pathway in vitro and induces protective immunity in vivo. Blood. 2004;104:1961-9.

33. Schiavo R, Baatar D, Olkhanud P, Indig F, Restifo N, Taub D, Biragyn A. Chemokine receptor targeting efficiently directs antigens to MHC class I pathways and elicits antigen-specific CD8+ T-cell responses. Blood. 2006; 107:4597-605.

34. Guo J, Fan M, Sun J, Jia R. Fusion of antigen to chemokine CCL20 or CXCL13 strategy to enhance DNA vaccine potency. Int Immunopharmacol. 2009;9:925930

35. Song R, Liu S, Leong KW. Effects of MIP-1 alpha, MIP-3 alpha, and MIP-3 beta on the induction of HIV Gag-specific immune response with DNA vaccines. Mol Ther. 2007;15:1007-15.

36. Oppenheim JJ, Biragyn A, Kwak LW, Yang D. Roles of antimicrobial peptides such as defensins in innate and adaptive immunity. Ann Rheum Dis. 2003; 62 Suppl 2:ii17-21.

37. Coscia M, Biragyn A. Cancer immunotherapy with chemoattractant peptides. Semin Cancer Biol. 2004;14:209218.

38. Biragyn A, Tani K, Grimm M, Weeks S, Kwak L. Genetic fusion of chemokines to a self tumor antigen induces protective, T-cell dependent antitumor immunity. Nat Biotechnol. 1999;17:253-8.

39. Biragyn A, Surenhu M, Yang D, Ruffini PA, Haines BA, Klyushnenkova E, Oppenheim JJ, Kwak LW. Mediators of innate immunity that target immature, but not mature, dendritic cells induce antitumor immunity when genetically fused with nonimmunogenic tumor antigens. J Immunol. 2001; 167:6644-53.

40. Ma K, Xu W, Shao X, Yanyue, Hu L, Xu H, Yuan Z, Zheng X, Xiong S. Coimmunization with RANTES plasmid polarized Th1 immune response against hepatitis $B$ virus envelope via recruitment of dendritic cells. Antiviral Res. 2007;76:140-9. 
41. Miguel A, Herrero M, Sendra L, Botella R, Algás R, Sánchez M, Aliño S. Comparative antitumor effect of preventive versus therapeutic vaccines employing B16 melanoma cells genetically modified to express GM-CSF and B7.2 in a Murine Model. Toxins. 2012;4:1058-81.

42. Yan J, Pankhong P, Shin TH, Obeng-Adjei N, Morrow MP, Walters JN, Khan AS, Sardesai NY, Weiner DB. Highly optimized DNA vaccine targeting human telomerase reverse transcriptase stimulates potent antitumor immunity. Cancer Immunol Res. 2013;1:179-89.

43. Tuthill RJ, Unger JM, Liu PY, Flaherty LE, Sondak VK. Risk assessment in localized primary cutaneous melanoma. Am J Clin Pathol. 2002;118:504-11.

44. Haabeth OA, Tveita AA, Fauskanger M, Schjesvold F, Lorvik KB, Hofgaard PO, Omholt H, Munthe LA, Dembic Z, Corthay A, Bogen B. How Do CD4(+) T cells detect and eliminate tumor cells that either lack or express MHC class II molecules? Front Immunol. 2014;5:174.

45. Igoucheva O, Grazzini M, Pidich A, Kemp DM, Larijani M, Farber M, Lorton J, Rodeck U, Alexeev V. Immunotargeting and eradication of orthotopic melanoma using a chemokine-enhanced DNA vaccine. Gene Ther. 2013;20: 939-48

46. Castle J, Kreiter S, Diekmann J, Löwer M, van de Roemer N, de Graaf J, Selmi A, Diken M, Boegel S, Paret C, Koslowski M, Kuhn A, Britten C, Huber C, Türeci Ö, Sahin U. Exploiting the mutanome for tumor vaccination. Cancer Res. 2012;72:1081-91.

\section{Submit your next manuscript to BioMed Central} and we will help you at every step:

- We accept pre-submission inquiries

- Our selector tool helps you to find the most relevant journal

- We provide round the clock customer support

- Convenient online submission

- Thorough peer review

- Inclusion in PubMed and all major indexing services

- Maximum visibility for your research

Submit your manuscript at www.biomedcentral.com/submit 